\title{
Long-term safety, efficacy, and patient acceptability of teriparatide in the management of glucocorticoid-induced osteoporosis
}

This article was published in the following Dove Press journal:

Patient Preference and Adherence

17 May 2013

Number of times this article has been viewed

\section{Robin K Dore}

David Geffen School of Medicine, University of California, Los Angeles, CA, USA
Correspondence: Robin K Dore David Geffen School of Medicine, University of California, 1279| Newport Ave \#20I, Tustin, CA 92780, USA

Email rkdmail@sbcglobal.net

\begin{abstract}
Glucocorticoids are commonly prescribed medications to treat multiple diseases across many medical specialties. One of the most common yet largely unappreciated side effect of glucocorticoid use is increased risk of fracture. Many different therapies are indicated to prevent and treat this condition; many guidelines exist that suggest appropriate use of both glucocorticoids and the medications approved to prevent this common side effect of glucocorticoid therapy. Nevertheless, $30 \%-50 \%$ of patients on long-term glucocorticoid therapy sustain a fracture. Teriparatide, recombinant human parathyroid hormone (1-34), is a daily self-injectable therapy for 24 months approved for use in patients taking long-term glucocorticoids. Teriparatide has been shown to increase bone mineral density and reduce vertebral fracture risk in glucocorticoidtreated patients. Glucocorticoids have many adverse effects on bone that teriparatide has been shown to prevent or negate. Given the fact that preventive therapy for glucocorticoid-induced osteoporosis is often not prescribed, one wonders whether a daily self-injectable therapy for this condition would be prescribed by physicians and accepted by patients. This article reviews the epidemiology, pathophysiology, treatment, guidelines, and persistence data (when available) for patients with glucocorticoid-induced osteoporosis treated with teriparatide.
\end{abstract}

Keywords: glucocorticoid-induced osteoporosis, teriparatide, anabolic, PTH, parathyroid hormone

\section{Introduction}

Glucocorticoids are widely prescribed medications to treat multiple diseases across many specialties in medicine. It is estimated that $2.5 \%$ of the elderly population, defined as 70-79 years of age, are prescribed oral glucocorticoid therapy. ${ }^{1}$ Glucocorticoids are used in $0.2 \%-0.5 \%$ of the general population. ${ }^{2}$ Due to glucocorticoids' extensive use, glucocorticoid therapy is the most common cause of secondary osteoporosis and the primary cause of drug-induced osteoporosis. ${ }^{3}$ Often, the presenting manifestation is a fracture, which occurs in $30 \%-50 \%$ of patients who receive long-term glucocorticoid therapy. ${ }^{4}$ The fracture risk increases with the daily dose rising from 1.55 for vertebral fractures at a daily dose of $2.5 \mathrm{mg}$ of prednisolone or less up to 5.18 at a daily dose of $7.5 \mathrm{mg}$ a day or more. These fractures may occur early after the initiation of therapy; the risk of fracture increases by as much as $75 \%$ within the first 3 months of therapy before there is a significant decline in bone mineral density (BMD). ${ }^{5}$ Bone loss is more pronounced in the trabecular bone that is found predominantly in the spine, ribs, and proximal femur. ${ }^{6}$ The bone loss is biphasic, with a rapid initial phase of $6 \%-12 \%$ loss in the first year of glucocorticoid therapy, followed by a slower phase during continued use of $0.5 \%-3 \%$ loss annually. ${ }^{7}$ 
Many guidelines have been published regarding the prevention and treatment of glucocorticoid-induced osteoporosis, including those from the American College of Rheumatology (ACR) and the National Institute for Health and Clinical Excellence. ${ }^{8,9}$ Unfortunately, only a small minority of glucocorticoid users receive effective preventive, diagnostic, and therapeutic interventions. ${ }^{10}$ Perhaps this lack of prevention is due to the misunderstanding that even small doses of glucocorticoids - or use of glucocorticoids for a short period of time increase the risk of fracture. ${ }^{11}$

Many medications are approved for the prevention (risedronate, zoledronic acid) and the treatment (alendronate, risedronate, zoledronic acid, teriparatide) ${ }^{12-15}$ of glucocorticoidinduced osteoporosis. All of the subjects in the clinical trials were treated with calcium and vitamin D; in most studies, if subjects had levels of 25-hydroxyvitamin D below $20 \mathrm{ng} / \mathrm{mL}$, treatment with vitamin D and subsequent rescreening was required to make certain that all patients had sufficient levels of vitamin D to participate in the trials. In the past, it was felt that glucocorticoids increased fracture risk due to an increase in bone resorption alone, and thus bisphosphonates that reduce osteoclast activity were felt to be first-line therapy for glucocorticoid-induced osteoporosis. Now, however, it is understood that the bone loss resulting from glucocorticoid use occurs through many different mechanisms (summarized in Table 1); therefore, teriparatide, a medication that increases bone formation, is now recommended for use in certain patient populations. ${ }^{16,17}$

Table I Glucocorticoid effects on bone

Direct effects on bone
Stimulates osteoclastogenesis
Decreases osteoblast function and life span
Increases osteoblast apoptosis
Impairs preosteoblast formation
Direct molecular effects
Blocks the stimulatory effect of insulin-like growth factor I on bone
formation
Opposes Wnt/beta-catenin signaling, resulting in decreased bone
formation
Affects stromal cell differentiation, shunting cell formation towards
more adipocyte formation
Increased levels of receptor activator of nuclear factor kappa B
ligand/macrophage colony-stimulating factor, resulting in more
osteoclastogenesis
Decreased levels of osteoprotegerin, resulting in increased
osteoclastogenesis and increased bone resorption
Decreased levels of estrogen, testosterone, and adrenal androgen levels
that have adverse effects on bone cells

Note: Data from Dore. ${ }^{17}$

\section{Glucocorticoid effects on bone cells Effects on osteoclasts}

Because glucocorticoid receptors are present on both osteoclasts and osteoblasts, bone tissue is a target for glucocorticoids. ${ }^{18}$ In addition, glucocorticoids induce different effects on bone depending on their concentration, length of therapy, and cell differentiation. ${ }^{19}$ The first effect induced by glucocorticoid therapy is rapid bone loss due to osteoclast activation. Short-term exposure to glucocorticoids results in an increase in the number and activity of osteoclasts, as well as a decrease in osteoclast apoptosis, leading to a net increase in the osteoclast life span. ${ }^{20}$ Glucocorticoids also regulate osteoclasts via osteoblast-mediated mechanisms by stimulating the synthesis of receptor activator of nuclear factor kappa B ligand (RANKL), which increases osteoclast precursors and eventually the total number of osteoclasts; stimulating the synthesis of granulocyte colony-stimulating factor which leads to osteoblast apoptosis; and by decreasing osteoprotegerin (OPG) expression, which is a natural inhibitor of RANKL, so less OPG results in more osteoclast precursors and more mature osteoclasts. ${ }^{21}$

\section{Effects on osteoblasts}

Long-term use or prescribing high dosages of glucocorticoids has an effect on osteoblast proliferation and survival of both osteoblasts and osteocytes; this apoptosis of osteoblasts and osteocytes occurs due to activation of caspase- 3 and activation of glycogen synthase kinase 3 beta, which plays a role in Wnt signaling. ${ }^{22,23}$ In the absence of glucocorticoids, Wnt binds to the low-density lipoprotein receptor-related proteins 5 and 6 and their coreceptor, frizzled, stabilizes $\beta$-catenin, which results in the transcription of target genes and subsequent induction of bone formation. Glucocorticoids have been shown to suppress this pathway by increasing the production of Wnt-pathway inhibitors, such as Dickkopf-1. ${ }^{24}$

\section{Effects on bone marrow stromal cells}

Glucocorticoids have also been shown to stimulate bone marrow stromal cells to differentiate toward adipocytes rather than osteoblasts. This is mediated primarily through an increased expression of the peroxisome proliferator-activated receptor-gamma 2(PPAR- $\gamma 2$ ) and repression of the osteogenic transcription factor runt-related protein $2 .{ }^{25}$ Recent data suggest that glucocorticoid receptor signaling in response to a high concentration of glucocorticoid supports adipogenesis but inhibits osteogenesis by reducing c-Jun expression and human bone marrow stromal cell proliferation. ${ }^{26}$ 


\section{Effects on osteocytes}

Many investigators feel that neither the changes in bone metabolism and architecture nor the modest amount of apoptosis of osteoblasts and osteocytes explain the elevated bone fragility seen in glucocorticoid-treated patients. ${ }^{1}$ These investigators have reported an increase in osteocyte lacunar size and loss of perilacunar mineral with glucocorticoid treatment; this suggests that the osteocyte is either metabolically stressed or compromised in some way. In this model, less than $5 \%$ of osteocytes were apoptotic, suggesting that another effect in addition to apoptosis might be playing a role. ${ }^{27}$ These authors have suggested that autophagy, a protective mechanism by which cells can respond to stress, may play a role in glucocorticoid-induced changes in osteocytes. Autophagy is a lysosomal degradation pathway that is essential for cell differentiation, development, growth, survival, and homeostasis; it helps maintain the balance among the synthesis, degradation, and recycling of cellular products. For example, during autophagy, parts of the cytoplasm and intracellular organelles are sequestered within autophagic vacuoles that are eventually delivered to lysosomes for degradation. ${ }^{28}$ Excessive autophagy can destroy cellular components, but appropriate autophagy can protect cells from apoptosis by removing oxidatively damaged organelles. Therefore, autophagy can be a selfdestructive process that leads to cell death (apoptosis) or can preserve cellular viability. With glucocorticoid treatment, initially autophagy was a protective mechanism against apoptosis; however, under higher levels of stress or prolonged stress, a large accumulation of autophagosomes may accumulate, leading to cell apoptosis. In a mouse model, apoptotic tunnel-positive osteocytes and osteoblasts were increased in vertebral trabecular bone treated with 56 days of prednisolone. Based on these data, long-term treatment with glucocorticoids leads to apoptosis, and subsequently a reduction in bone strength and bone loss. ${ }^{29}$

\section{Teriparatide in glucocorticoid- induced osteoporosis Effects on bone cells}

Theoretically, an anabolic agent should be the primary therapy in treating glucocorticoid-induced osteoporosis, based on the glucocorticoid effects on bone reviewed above. The beneficial effects of daily injection of human parathyroid hormone ( $\mathrm{hPTH}$ ) amino-terminal peptide 1-34 have been demonstrated in men and women with glucocorticoid-induced osteoporosis. ${ }^{30}$ Osteoblasts, bone-lining cells and bone marrow stromal cells have PTH receptors; daily injections of teriparatide stimulate these cells through the modulation of cyclic adenosine monophosphate concentrations and cyclic adenosine monophosphate-dependent protein kinase $\mathrm{A} .{ }^{31}$ The PTH receptor also activates the calcium protein kinase $\mathrm{C}$ pathway, thus stimulating proliferation of cells in the osteoblastic lineage. ${ }^{32}$ Teriparatide produces some of its anabolic effect by stimulating the mitogenactivated protein kinase pathway, resulting in modulation of the differentiation, proliferation, and activity of the osteoblast pool. ${ }^{33,34}$ Teriparatide also has been shown to stimulate the differentiation and activation of quiescent bone-lining cells and increase the life span of osteoblasts and osteocytes by inhibiting their apoptosis. ${ }^{35,36}$ Some of these effects appear to be mediated by the direct activation of the early transcription factor Runx2, which plays an important role in osteoblast differentiation, perhaps by activating a protein kinase A-dependent increase and/or modulating the expression of cyclins and cyclin-dependent kinase inhibitors that are important in the survival and differentiating effects of PTH on cells of the osteoblastic lineage. ${ }^{37}$ Teriparatide also controls replication, differentiation, and survival of osteoblast precursors by influencing the synthesis and release of mediators such as insulin-like growth factor 1, fibroblast growth factor 2 , interleukin 6 (IL-6), Wnt, bone morphogenic protein, and transforming growth factor beta. ${ }^{38}$

Teriparatide has been shown to stimulate bone levels of insulin-like growth factor 1 that have been decreased by glucocorticoids, resulting in more osteoblast precursors and greater osteoblast survival. ${ }^{35}$ Teriparatide also has been shown to modulate both Wnt and sclerostin expression, which play opposite roles in bone formation. ${ }^{39}$ Teriparatide blunts mRNA and protein expression of sclerostin, a secreted osteocytic factor that prevents the binding of Wnt ligands to their receptors and subsequent bone formation. Finally, teriparatide inhibits PPAR- $\gamma$ transactivation activity, resulting in a decrease in adipocyte differentiation and increasing the number of osteoblasts. ${ }^{40}$ Thus, teriparatide antagonizes many of the deleterious effects of glucocorticoids on bone (Table 2). ${ }^{41}$

\section{Clinical trial data}

Subcutaneous teriparatide $20 \mathrm{mcg}$ was compared to oral alendronate $10 \mathrm{mg}$ daily in 428 men and women with osteoporosis who had received glucocorticoids at a dose of $5 \mathrm{mg}$ per day or more for at least 3 months in a double-blind, randomized, controlled clinical trial. ${ }^{30}$ The primary outcome was the change from baseline to 18 months in BMD at the 


\section{Table 2 Teriparatide effects on bone}

Increases levels of insulin-like growth factor I, resulting in increases in the number of osteoblast precursors, and increases osteoblast survival Activates the calcium protein kinase $C$ pathway that stimulates proliferation of cells in the osteoblastic lineage Increases the expression of Wnt signaling, resulting in increased bone formation

Blunts the mRNA and protein expression of sclerostin, a Wnt antagonist, resulting in increased bone formation Controls the replication and differentiation and survival of osteoblast precursors by influencing the expression and release of fibroblast growth factor 2 , interleukin 6 , bone morphogenetic protein, and transforming growth factor beta

Activates quiescent bone-lining cells

Increases trabecular bone volume

Decreases osteoblast apoptosis

Increases bone strength (in animal models)

Note: Data from Yao et al. .1 $^{41}$

lumbar spine. Prespecified secondary outcomes included the incidence of vertebral and nonvertebral fractures and adverse events. Subjects were enrolled in the trial if the T-score at the lumbar spine or total hip was -2.0 or less or the T-score was -1.0 or less with at least one fragility fracture occurring during treatment with glucocorticoids. After 18 months, the BMD in the lumbar spine increased significantly more in the teriparatide-treated patients than in the alendronate-treated patients $(7.2 \%$ vs $3.4 \%)$; a significant difference between the two groups was reached by 6 months $(P<0.001)$. At 12 months, the BMD at the total hip had increased more in the teriparatide group. A difference in the number of patients with new vertebral fractures was observed as well $(0.6 \%$ vs $6.1 \%, P=0.004)$, but the study was not statistically powered to assess a reduction in the risk of vertebral fracture; the validity of these data was also limited as paired radiographs (baseline and postbaseline) for the assessment of new vertebral fractures were missing for 92 patients. No difference was seen with regard to a reduction in nonvertebral fractures $(5.6 \%$ vs $3.7 \%, P=0.36)$. Significantly more patients in the teriparatide group had at least one elevated serum calcium level.

A post hoc analysis evaluated the effect of baseline glucocorticoid dose on the 18-month BMD response to teriparatide or alendronate..$^{42}$ Mean baseline glucocorticoid doses were categorized as low dose ( $\leq 5 \mathrm{mg}$ /day), medium dose ( $>5$ and $<15 \mathrm{mg} /$ day), and high dose ( $\geq 15 \mathrm{mg} /$ day). Baseline lumbar spine, femoral neck, and total hip BMD values were similar between groups and between the glucocorticoid dosages within each group. Lumbar spine BMD increases at the low, medium, and high glucocorticoid doses were $8.1 \%, 6.6 \%$, and $4.6 \%$, respectively, with teriparatide and $3.6 \%, 2.8 \%$, and $2.3 \%$ with alendronate.
Glucocorticoid dose did not have a statistically significant effect on femoral neck or total hip BMD changes in either group.

A follow-up study by Saag et al reported the 36-month results of this study. ${ }^{43}$ At 36 months, the increases in the lumbar spine BMD comparing teriparatide and alendronate were $11.0 \%$ versus $5.3 \%$ in the lumbar spine, $5.2 \%$ versus $2.7 \%$ in the total hip, and $6.3 \%$ versus $3.4 \%$ in femoral neck $(P<0.001)$. Fewer subjects had vertebral fractures in the teriparatide group than in the alendronate group: $1.7 \%(3 / 173)$ versus $7.7 \%(13 / 169) ; P=0.007$. There continued to be no significant difference between the two groups in the incidence of nonvertebral fractures between teriparatide and alendronate, with 7.5\% (16/124) versus 7.7\% (15/214), $P=0.843$. As in the 18 -month part of the study, elevated serum calcium levels were seen more frequently in the teriparatide subjects: $21 \%$ versus $7 \%(P<0.001)$.

Langsdahl et al performed an additional analysis of this data by sex and menopausal status. ${ }^{44}$ At 18 months, the lumbar spine BMD increases were significantly greater in the teriparatide versus alendronate group in men $(7.3 \%$ versus $3.7 \%, P=0.03)$, premenopausal women $(7.0 \%$ versus $0.7 \%, P<0.001)$, and postmenopausal women $(7.8 \%$ versus $3.7 \%, P<0.001)$. Morphometric vertebral fractures occurred in one teriparatide (postmenopausal woman) and ten alendronate subjects (six postmenopausal women, four men). Nonvertebral fractures occurred in twelve teriparatide (nine postmenopausal women, two premenopausal women, and one man) and eight alendronate subjects (six postmenopausal women and two men). Adverse events were evenly distributed among the subgroups.

Losada et al published another post hoc analysis comparing Hispanic $(\mathrm{n}=61)$ and non-Hispanic cohorts ( $\mathrm{n}=367) .{ }^{45}$ At 18 months in the Hispanic cohort, there were significantly greater increases from baseline in the teriparatide subjects versus alendronate subjects in total BMD $(5.9 \%$ versus $1.3 \%, P<0.001)$ and lumbar spine BMD $(9.8 \%$ versus $4.2 \%, P<0.001)$; there was not a significant difference between groups at the femoral neck. There was not a statistically significant difference in BMD within each treatment group between the Hispanic versus the non-Hispanic cohort. The number of subjects reporting one or more adverse events was not significantly different between treatment groups in either cohort, but more subjects in the teriparatide group reported nausea.

In another study involving $400 \mathrm{U}$ of hPTH (1-34), 51 subjects with glucocorticoid-induced osteoporosis were randomly assigned to receive this form of PTH plus hormone-replacement therapy versus hormone-replacement 
therapy alone. ${ }^{21}$ Serum levels of soluble RANKL, OPG, IL-6, and IL-6 soluble receptor were measured at baseline, 1 month, and every 3 months thereafter for a total of 24 months. Treatment with PTH was associated with a significant increase in soluble RANKL within 1 month, and these levels remained elevated throughout the length of the study. The levels of IL-6 and IL-6 soluble receptor increased significantly within 1 month, but returned to baseline levels. OPG levels, in contrast, were mildly suppressed beginning at 6 months of hPTH therapy. These data support the hypothesis that hPTH (1-34) stimulates osteoblast maturation and function initially; these osteoblasts then express RANKL, leading to osteoclast activation and an eventual balance of bone formation and resorption.

Gluer et al published data comparing the effects of teriparatide and risedronate in glucocorticoid-induced osteoporosis in men. ${ }^{46}$ This study was an 18 -month, randomized, open-label, controlled trial enrolling male subjects who had taken glucocorticoids for more than 3 months at a dose of more than $5 \mathrm{mg}$ of prednisone per day and had an areal BMD T-score of -1.5 or less. Subjects were randomized to either $20 \mathrm{mcg}$ of teriparatide daily or risedronate $35 \mathrm{mg} 1$ day per week. The primary end point was the increase in L1-3 spine volumetric BMD between groups, as measured by quantitative computed tomography (QCT). Secondary end points included changes in 3-D microstructure variables, as measured by high-resolution QCT at T12, biomechanical effects, as measured by finite element analysis, areal BMD, as measured by dual-energy absorptiometry (DXA), and markers of bone turnover and safety. Imaging studies were performed at baseline and 6 and 18 months. Subjects entered in the study had a mean age of 56 years, median glucocorticoid daily dose of $8.8 \mathrm{mg}$, and duration of use of 6.4 years. A total of $39.1 \%(n=36)$ of the randomized subjects had a prevalent vertebral fracture, and $32.6 \%(n=30)$ had used bisphosphonates in the past. Results of the study revealed that both treatments increased the volumetric BMD, with greater increases in the teriparatide group $(17.1 \%$ versus $5.3 \%, P=0.004)$. There were significant increases in microstructure-derived high-resolution QCT trabecular and cortical variables from baseline in both groups, but the differences were not statistically significant. Vertebral strength and stiffness increased significantly in both treatment groups, with statistically greater increases in the teriparatide treatment arm for all variables measured. There was a trend for higher bone volume/trabecular volume in the teriparatide-treated subjects, but this did not reach statistical significance $(23.1 \%$ versus $7.3 \%, P=0.074)$. Fewer subjects in the teriparatide group had a new clinical fracture compared with risedronate ( $0 / 45$ versus $5 / 47, P=0.056)$. Reported adverse events were not different between the two treatment groups.

\section{Guidelines for the prevention and treatment of glucocorticoid- induced osteoporosis American College of Rheumatology guidelines}

The ACR updated their guidelines in 2010 , given the approval of two new medications for the treatment of glucocorticoidinduced osteoporosis, one of which was teriparatide, and the understanding that bone density alone did not accurately predict fracture risk. ${ }^{12}$ The recommendations in the guidelines were developed based using the Rand/UCLA appropriateness method, which consisted of a systematic review of research papers published between January 1966 and August 28, 2008 and the subsequent work of a core executive panel with the assistance of two expert panels: the Expert/Advisory Panel, which framed the development of the recommendations, and the Task Force Panel, which voted on the specific recommendations. Treatment recommendations were based on defining patients as low-, medium-, or high-risk either by using the fracture risk assessment (FRAX) tool or the reliance of clinicians on examples of patients that were typical of low-, medium-, and high-risk categories, and examples of each were included in the guidelines. ${ }^{47}$

Using the FRAX calculation, the Expert Advisory Panel defined a 10-year risk of major osteoporotic fracture of $10 \%$ or less as low-risk, $10 \%-20 \%$ as medium-risk, and greater than a $20 \%$ risk, a T-score of -2.5 or lower, or a history of a fragility fracture as high-risk. As FRAX uses an average glucocorticoid dose to calculate the 10-year fracture risk, patients who receive higher daily doses of glucocorticoids, higher cumulative glucocorticoid doses, or intravenous glucocorticoids will most likely have a greater absolute fracture risk than using the method outlined above. It is suggested that these additional factors be taken into consideration in determining a patient's fracture risk and perhaps shifting the risk into a higher category.

The recommended approach to postmenopausal women and men above the age of 50 years initiating or receiving glucocorticoid therapy who are in the high-risk group is to consider teriparatide if any dose of glucocorticoids is to be used for longer than 1 month (alendronate, risedronate, and zoledronic acid are also included in the recommendations 
for this group of patients). The recommendations become more complicated for premenopausal women and men below the age of 50 years initiating or receiving glucocorticoid treatment. The recommendations for premenopausal women of nonchildbearing potential and men below the age of 50 are similar: teriparatide is recommended if a fragility fracture is present, and glucocorticoid therapy is to be used for more than 3 months (alendronate, risedronate, and zoledronic acid are also recommended therapies for these two groups). For premenopausal women with childbearing potential who have a prevalent fragility fracture, teriparatide is recommended if glucocorticoids are prescribed for more than 3 months at a dose of $7.5 \mathrm{mg}$ or more of prednisone (alendronate and risedronate are also recommended for this group). A caveat included in the guidelines states that "recommendations for premenopausal women and younger men are constrained by the paucity of evidence for fracture risk and the treatment of GIOP in this population." Another limitation of this model is that FRAX only utilizes the hip BMD in calculating fracture risk. This can be an issue in glucocorticoid-induced osteoporosis, as bone loss typically occurs in trabecular bone found in the spine before bone loss occurs in cortical bone found in the hip, and vertebral fractures occur more frequently and earlier during the course of glucocorticoid therapy. Thus, using FRAX may underestimate fracture risk, especially early on during glucocorticoid therapy. However, despite these caveats, these guidelines do incorporate a more comprehensive, updated approach to the prevention and treatment of glucocorticoid-induced osteoporosis, although some practitioners find them too complex to be used in the clinical setting.

\section{American Society of Bone and Mineral Research recommendations}

Subsequently, the American Society of Bone and Mineral Research (ASBMR) published an article that presented examples of patient scenarios for which this group felt that treatment was uncertain based on the ACR guidelines or disagreed with the ACR interpretation of published data and presented their own treatment recommendations. ${ }^{48}$ The ASBMR committee concluded that existing data did not support ranking the evidence on glucocorticoidinduced osteoporosis treatment efficacy for alendronate higher than teriparatide. This conclusion was based on the lack of "convincing" fracture data for alendronate and "compelling" data from the head-to-head clinical trial of teriparatide versus alendronate, which demonstrated that teriparatide was statistically superior to alendronate both in bone density increases and vertebral fracture reduction. ${ }^{30}$ As the glucocorticoid-induced osteoporosis trials were of short duration - risedronate and zoledronic acid for 1 year, alendronate for 2 years, and teriparatide for 3 years - and patients take glucocorticoids often for many years, the ASBMR committee noted that there are essentially no data on the long-term safety or efficacy of bisphosphonate or teriparatide therapy in these patients. Therefore, the committee recommended that patients who require longterm glucocorticoid therapy be evaluated for substitution of bisphosphonate therapy after 1-2 years with teriparatide or be considered for initial therapy with teriparatide followed by treatment with a bisphosphonate. The ASBMR committee also recommended that postmenopausal women and men over the age of 50 years who are in the high-risk fracture group be considered for teriparatide therapy independent of glucocorticoid dose or duration based on the entry criteria for the head-to-head teriparatide versus alendronate trial. ${ }^{31}$ This committee also suggested that teriparatide be considered as initial therapy for women considering future pregnancy and who have closed epiphyses, as teriparatide, unlike bisphosphonates, does not have skeletal retention when therapy is stopped; therefore, if a woman becomes pregnant while taking teriparatide, it is not expected to affect fetal bone growth. In the head-to-head trial of teriparatide versus alendronate, neither therapy was found to reduce fractures in premenopausal women, but women assigned to the teriparatide group had greater increases in BMD at the lumbar spine and total hip at 18 months (7\% versus $0.7 \%$, $P<0.001) .{ }^{30}$ The ASBMR committee concluded that since the bone density response to teriparatide in this trial was similar for premenopausal and postmenopausal women, the response to teriparatide therapy was independent of baseline bone density, bone turnover or menopausal status, and thus they concluded that teriparatide should be a "reasonable" treatment option for premenopausal women who plan a future pregnancy.

\section{Adherence to guidelines}

Unfortunately, adherence to the recommendations and guidelines for the treatment and prevention of glucocorticoid-induced osteoporosis has been low. ${ }^{10}$ This was demonstrated for the 2001 ACR guidelines, but has not been studied yet for the 2010 ACR guidelines due to their recent publication. ${ }^{49}$ It is not expected, however, that adherence to these guidelines will be any greater due to their complexity and the fact that the use of FRAX or referring to clinical scenarios is required to establish 
whether the patient is in a low-, medium- or high-risk fracture category.

\section{Patient scenarios}

A 54-year-old African-American female was recently diagnosed with systemic lupus; she is postmenopausal. Therapy with glucocorticoids was initiated at a dose of $10 \mathrm{mg}$ /day. Her DXA revealed a T-score of -2.1 in the lumbar spine and -1.4 in the femoral neck. She denies any prior fractures. Her FRAX score for 10-year risk of osteoporotic fracture is 4.4. Preventive therapy with weekly alendronate was initiated. As per the ACR guidelines, her FRAX score places her in the low-risk group and non-teriparatide therapy is advised.

A 74-year-old Chinese female was recently diagnosed with giant-cell arteritis. She has a history of two prevalent vertebral fractures. Therapy with glucocorticoids was initiated at a dose of $30 \mathrm{mg} /$ day. Her DXA revealed a T-score in the femoral neck of -2.6. Her FRAX score for 10-year risk of major osteoporotic fracture is 22. Preventive therapy with daily teriparatide was initiated. As per the ACR guidelines, her FRAX score places her in the high-risk group, as does her T-score of -2.6 and her history of prior fractures; therefore, teriparatide is an option.

No guidelines exist for monitoring response to teriparatide therapy in patients with glucocorticoid-induced osteoporosis. My practice is to obtain a DXA at baseline to determine the fracture risk and to perform a second DXA at the end of the 2 years of therapy, which acts as a baseline for future therapy. In my own practice, I do not follow markers of bone turnover or monitor BMD more frequently, as these results can be affected by prior osteoporosis therapy and individual response to teriparatide.

Although the study by Saag et $\mathrm{al}^{43}$ evaluated 36 months of teriparatide treatment in patients with glucocorticoidinduced osteoporosis, at present teriparatide is only approved for a maximum of 24 months of therapy. Many patients who complete 2 years of teriparatide therapy are still receiving glucocorticoid therapy. It is common practice to begin antiresorptive therapy with either bisphosphonates or denosumab (off-label use, as denosumab is not approved for use in glucocorticoid-induced osteoporosis) after completing 2 years of teriparatide to maintain the bone density increases and presumed structural improvements in bone; no randomized controlled trials have investigated this topic. It is not usually recommended to take a "drug holiday" from bisphosphonate therapy if a patient is on prolonged glucocorticoid therapy, although this issue is controversial. No safety data have been published regarding retreatment with teriparatide once the 2-year course of therapy has been completed.

\section{Adherence to teriparatide therapy in the treatment of glucocorticoid- induced osteoporosis Direct analysis of nonvertebral fractures in the DANCE study}

Little has been published about the adherence to and persistence with therapy with teriparatide in glucocorticoidinduced osteoporosis. The data concerning the adherence and persistence to therapy with teriparatide in severe osteoporosis will be reviewed, and comments will be made as to how these data do or do not pertain to patients with glucocorticoidinduced osteoporosis. The largest study to date is based on questionnaires that were completed at a subset of sites participating in the Direct Analysis of Nonvertebral Fractures in the Community Experience (DANCE) study, which was a prospective, observational trial designed to examine the efficacy and tolerability of teriparatide as used in clinical practice (ClinicalTrials.gov identifier: NCT01078805). ${ }^{50,51}$ This multicenter, multispecialty trial enrolled subjects who were treated with teriparatide, some of whom were taking glucocorticoids. The subjects were given a baseline questionnaire that included items related to the subject's prior experience with self-injection of drugs, perception as to the severity of their osteoporosis, relationship with the prescribing health-care professional and his/her staff, and concerns about starting teriparatide therapy. Subjects who discontinued teriparatide between baseline and 12 months were asked to complete a questionnaire that addressed possible reasons for discontinuation. Subjects who did not report discontinuation were considered as persistent.

At study initiation, most of the subjects felt that their osteoporosis was "severe" or "very severe" ( $n=746,59 \%)$. Almost all of the subjects felt that it was "very important" or "extremely important" to treat their osteoporosis $(n=1217$, $96 \%$ ). Most of the subjects felt that teriparatide would be "very effective" or "extremely effective" in reducing the risk of fracture $(n=1064,84 \%)$. Some subjects were "very concerned" or "extremely concerned" about their ability to pay for this therapy $(n=374,30 \%)$. Twenty-five percent of subjects reported side effects $(n=234)$ with approximately half of this group $(n=120)$ reporting "moderate" or "severe" side effects. At month $12,15 \%$ of the remaining subjects reported experiencing side effects. Early discontinuation between months 2 and 6 and late discontinuation between 
months 6 and 12 were frequently due to financial reasons or concerns about treatment side effects outweighing treatment benefits. There was also an association between early discontinuation and the perception that the prescribing health-care professional had a low level of knowledge about teriparatide $(P=0.01)$, low enthusiasm for continued treatment with teriparatide $(P=0.01)$, or a low level of confidence in therapy with teriparatide $(P<0.001)$. Side effects were significantly associated with both early and late discontinuation. A subject not perceiving the importance of continuing treatment was associated with late discontinuation. A limitation of this study is the fact that it is not known whether the subjects in this study were representative of the typical patient who is prescribed teriparatide, as many of the investigators in the study were considered to be osteoporosis "experts" who perhaps had more baseline experience in prescribing teriparatide.

The findings of this study, however, are supported by a model developed by Brod et al that evaluated persistence and adherence with injectable drugs based on semistructured interviews of patients and physicians who received/ prescribed teriparatide. ${ }^{52}$ This model demonstrated that persistence and adherence with teriparatide injections were continuing processes that were influenced by both patient and health-care professional factors and that the process began even before therapy was initiated. I would surmise that this model should also apply to the use of teriparatide in patients with glucocorticoid osteoporosis, ie, the prescriber needs to explain to the patient the fact that they are at increased risk of fracture due to the use of glucocorticoids and that in the opinion of the prescriber, the benefits of teriparatide in reducing fracture outweigh the risks of both teriparatide and glucocorticoid therapy.

\section{Additional observational studies of teriparatide in glucocorticoid- induced osteoporosis}

A review of published data reveals many observational reports of the efficacy, safety, and adherence to treatment with teriparatide in different populations. Franceschetti et al present data on 135 Italian women treated with teriparatide for 18 months between 2005 and 2011. ${ }^{53}$ Treatment discontinuation due to adverse events was low, at $2.22 \%$ ( $1.48 \%$ for dyspnea, $0.74 \%$ for nausea and myalgia). Adherence and compliance were high, at 94.07\%, with eight subjects stopping treatment (5.93\%) and only three of these due to an adverse reaction. These authors measured efficacy as the incidence of new fractures that was low at
$0.74 \%$. A reduction in back pain, as evaluated by using a visual analog scale showed a significant reduction at the end of the study (visual analog score at baseline of 9.2 versus 4.2 at study termination, $P<0.0001)$.

Ziller et al evaluated the adherence and persistence in 50 postmenopausal women with severe osteoporosis treated with teriparatide. ${ }^{54}$ Apart from a significantly lower age at menopause in the adherent group (46.1 versus 50.0 years, $P<0.006$ ), there were no significant differences between the baseline characteristics of the adherent versus nonadherent subjects. At 12 months, $80 \%$ of the subjects treated with teriparatide were adherent; adherence was correlated to self-reported medication tolerability $(P<0.001)$. Unlike the DANCE trial, ability to afford medication was not a consideration in this trial.

Hazel-Fernandez et al evaluated the association of teriparatide adherence and persistence with clinical and economic outcomes in a Medicare Part D population. ${ }^{55}$ This was a retrospective cohort study that assessed medical and pharmacy claims for 761 Medicare members that were initiating teriparatide therapy in 2008 and 2009. The results of the study showed that within 12 months of teriparatide initiation, only $21 \%$ of the cohort was highly adherent, as measured by the proportion of days covered (PDC). Adherence was categorized as high if the PDC was $\geq 80 \%$, moderate $(50 \% \leq$ PDC $<80 \%)$, and low (PDC $<50 \%$ ). Low-adherent or nonpersistent patients visited the emergency room more frequently than did the highly adherent patients $\left(\chi^{2}=5.01, P<0.05\right.$ and $\chi^{2}=5.84$, $P<0.05)$. The low-adherent and nonpersistent groups also had significantly lower mean pharmacy costs $(\$ 4361$ versus $\$ 13,472$ and $\$ 4757$ versus $\$ 13,187, P<0.0001$ ). Nonpersistent patients had significantly lower total healthcare costs; the health-care costs of the highly adherent patients were primarily pharmacy-related. Regression models showed no significant association of adherence or persistence with 12-month fractures. Six months prior to initiating teriparatide therapy, $50.7 \%$ of the cohort had experienced at least one fracture. By 12 months, these fracture patients were almost three times more likely to have another fracture (odds ratio 2.9, 95\% confidence interval 2.1-4.1; $P<0.0001)$.

Foster et al evaluated the adherence and persistence with teriparatide therapy among patients with commercial, Medicare, and Medicaid insurance. ${ }^{56}$ This study evaluated beneficiaries with at least one claim for teriparatide in 2003 or 2004 that had continuous enrollment in the prior 12 months and subsequent 6 months in a national commercial/ 
Medicaid and Medicare administrative database (MarketScan). Adherence was assessed by calculating medication possession ratio. Persistence was assessed by measuring the time from initiation to discontinuation and time until first 60-day gap in therapy. Factors that were associated with persistence were assessed using a Cox proportional hazard model. The average medication possession ratio at 6 months was 0.74 $(\mathrm{n}=2218)$ and at twelve months was $0.66(\mathrm{n}=1303)$. At 6 months, $64.6 \%$ of patients remained on therapy, and $56.7 \%$ remained on therapy at 12 months. Lower patient copays, DXA screening, and use of antiresorptive therapy within the 12 months prior to initiation of teriparatide were associated with increased persistence. Data concerning persistence and adherence for glucocorticoid-induced osteoporosis were not assessed in this study.

Halpern et al reviewed the association of adherence to osteoporosis therapies, including teriparatide with fracture, all-cause medical costs, and all-cause medical costs. ${ }^{57}$ Not surprisingly, their data revealed that low adherence to osteoporosis therapy, including alendronate, ibandronate, risedronate, raloxifene, and teriparatide, was associated with a significantly increased risk of fracture. Unfortunately, data were not presented for the individual therapies and were not specific for glucocorticoid-induced osteoporosis.

\section{Efforts to improve adherence and persistence of therapy for glucocorticoid-induced osteoporosis}

Unfortunately, only a small minority of glucocorticoid users receive effective preventive, diagnostic, and therapeutic interventions. ${ }^{2}$ Solomon et al examined electronic medical records for patients with rheumatoid arthritis with an index visit in January or February of 1999 at a large rheumatology practice in an academic medical center. ${ }^{58}$ Of 539 eligible rheumatoid arthritis patients, 236 (44\%) were taking glucocorticoids. Of this group, only $23 \%$ had a DXA performed, but $42 \%$ were prescribed a medication to treat/prevent glucocorticoidinduced osteoporosis. Unfortunately, men and premenopausal women were less likely to be treated. Attempts to improve the number of patients treated by educating them have not been effective, nor have attempts at educating health-care professionals. ${ }^{11,59,60}$ Thus, the Geisinger Medical Center Department of Rheumatology developed the GlucocorticoidInduced Osteoporosis Program. ${ }^{61}$ The goal of this program was to identify patients at increased risk of fracture, provide education, redesign and implement new pathways of care, and to monitor outcomes. Two hundred chronic glucocorticoid- treated patients were seen at baseline, and follow up was performed at 6 and 12 months. Patients entered the study based on self-referral, physician referral, and a database search of glucocorticoid users. The study participants were assigned to either the consult-only arm or the consult/treat arm by their referring physician. The consult-only arm consisted of prevention, diagnosis, and treatment recommendations. In the consult/treat arm, in addition to what was performed in the consult-only arm, the participants also received education and follow-up. Standardized consult and follow-up communication templates were developed for the electronic health record. At the follow-up visits for the consult/treat arm, the participants underwent posteducation testing, review of their exercise program, 25-hydroxyvitamin D level was drawn, medication costs and glucocorticoid dose were recorded, and medication adherence was evaluated. Follow-up DXAs were performed at an interval specified by the protocol. At study end, the two groups were combined to monitor outcomes, so individual results for the two different arms are not available.

At the time of data analysis, 140 patients had completed their 6-month evaluation, and 83 patients had completed their 1 -year evaluation. Only 16 patients left the program, five of whom had died. Patient knowledge retention, frequency of exercise, and 25-hydroxyvitamin D levels were all improved significantly at 1 year. A significant decrease in glucocorticoid dose was also seen. In terms of treatment, $91 \%(49 / 54)$ of patients classified as at high risk of fracture were still either taking a bisphosphonate or teriparatide at 1 year. Reasons for not being on medication at 1 year included cost $(5 \%)$, an intercurrent health problem $(2 \%)$, and unknown (2\%). Bone density at the hip and spine also increased significantly. The authors suggest that the design of this program can and should be adapted by other health systems and organizations.

The manufacturer of teriparatide, Eli Lilly, has developed a program to provide patient support. ${ }^{62}$ This program provides support for injection training, a follow-up call 1 week after the injection training, and ongoing support with regard to reimbursement, questions concerning possible medication side effects, and reminders how to inject properly and continuing use of the medication. Patients must opt in to this program. Unfortunately, no data are available with regard to the impact of this program on patient adherence and compliance regarding teriparatide use for postmenopausal osteoporosis, male osteoporosis, or glucocorticoid-induced osteoporosis (Kathleen Taylor, Eli Lilly and Company, personal communication, February, 2013). 


\section{Conclusion}

Based on the pathogenesis of glucocorticoid-induced osteoporosis, anabolic agents such as teriparatide should be the ideal choice in glucocorticoid-treated patients. Nevertheless, no guidelines exist that suggest teriparatide as first-line therapy for glucocorticoid-induced osteoporosis, although the ACR guidelines do suggest its use in patients who are at very high risk of fracture, defined as a 10-year absolute fracture risk of greater than $20 \%$. This lack of recommendation of teriparatide in the treatment of glucocorticoid-induced osteoporosis is most likely based on cost, absence of longterm data due to the restricted duration of administration, and the actual or perceived inconvenience of a daily injection for 24 months. I agree with Teitelbaum et al, who suggest that comparative-effectiveness studies are urgently needed to better understand the benefits of antiresorptive and anabolic agents with regard to reduction in vertebral and nonvertebral fractures in the long-term management of glucocorticoid-induced osteoporosis.$^{63}$ Once these data are available, health-care professionals need to utilize them in choosing an appropriate therapy for their patients, and methods need to be implemented that encourage patient compliance and adherence. Until these data are available, hopefully a better understanding of the pathophysiology of glucocorticoid-induced osteoporosis and the mechanisms of action of teriparatide and antiresorptive therapy in treating this condition will result in appropriate use of these therapies to prevent future fractures.

\section{Disclosure}

Dr Dore has received consulting fees from Amgen, Eli Lilly, Merck and Novartis. She is on the speakers' bureau for Amgen, Eli Lilly, Novartis and Warner-Chilcott. She has received funding for clinical trial research from Amgen and Eli Lilly.

\section{References}

1. van Staa TP, Leufkens HG, Abenhaim L, Begaud B, Zhang B, Cooper C. Use of oral corticosteroids in the United Kingdom. QJM. 2000;93: 105-111.

2. Saag K. Glucocorticoid-induced osteoporosis. Endocrinol Metab Clin North Am. 2003;32:135-157.

3. Canalis E, Mazziotti G, Giustina A, Bilezikian JP. Glucocorticoidinduced osteoporosis: pathophysiology and therapy. Osteoporos Int. 2007;18:1319-1328.

4. Adler RA, Curtis JR, Weinstein RS, Saag K. Glucocorticoid-induced osteoporosis. In: Feldman D, Marcus R, Nelson D, Rosen CJ. Osteoporosis, 3rd ed. San Diego: Elsevier-Academic Press; 2008:1135-1166.

5. Van Staa TP, Laan RF, Barton IP, Cohen S, Reid DM, Cooper C. Bone density threshold and other predictors of vertebral fracture in patients receiving oral glucocorticoid therapy. Arthritis Rheum. 2003;48: 3224-3229.
6. Van Staa TP, Leufkens HG, Cooper C. The epidemiology of corticosteroid-induced osteoporosis. a meta-analysis. Osteoporos Int. 2002;13:777-787.

7. LoCascio V, Bonucci E, Imbimbo B, et al. Bone loss in response to long-term glucocorticoid therapy. Bone Miner. 1990;8:39-51.

8. Grossman JM, Gordon R, Ranganath VK, et al. American College of Rheumatology 2010 Recommendations for the Prevention and Treatment of Glucocorticoid-Induced Osteoporosis. Arthritis Care Res (Hoboken). 2010;62:1515-1526.

9. Bone and Tooth Society of Great Britain, National Osteoporosis Society, Royal College of Physicians. Glucocorticoid-Induced Osteoporosis: Guidelines for Prevention and Treatment. London: Royal College of Physicians; 2002. Available from: http://bookshop.rcplondon.ac.uk/ contents/966c62dd-8011-4f65-a61d-dd0c7fe4fa4b.pdf. Accessed February 21, 2013.

10. Curtis JR, Westfall AO, Allison LL, et al. Longitudinal patterns in the prevention of osteoporosis in glucocorticoid-treated patients. Arthritis Rheum. 2005;52:2485-2494.

11. Blalock SJ, Norton LL, Patel RA, Dooley MA. Patient knowledge, beliefs and behavior concerning the prevention and treatment of glucocorticoidinduced osteoporosis. Arthritis Rheum. 2005;53:732-739.

12. Warner-Chilcott. Actonel (risedronate) [package insert]. Rockaway (NJ): Warner-Chilcott.

13. Novartis Pharmaceuticals. Reclast (zoledronic acid) [package insert]. East Hanover (NJ): Novartis Pharmaceuticals.

14. Merck. Fosamax (alendronate) [package insert]. Whitehouse Station (NJ): Merck.

15. Eli Lilly. Forteo (teriparatide) package insert. Indianapolis: Eli Lilly.

16. Canalis E, Bilezikian JP, Angeli A, et al. Perspectives on glucocorticoidinduced osteoporosis. Bone. 2004;34:593-598.

17. Dore RK. How to prevent glucocorticoid-induced osteoporosis. Cleve Clin J Med. 2010;77:529-536.

18. Beavan S, Horner A, Bord S, Ireland D, Compston J. Colocalization of glucocorticoid and mineralocorticoid receptors in human bone. J Bone Miner Res. 2001;16:1496-1504.

19. Cooper MS, Hewison M, Steward PM. Glucocorticoid activity, inactivity and the osteoblast. J Endocrinol. 1999;163:159-164.

20. Weinstein RS. Glucocorticoid-induced osteoporosis. Rev Endocr Metab Disord. 2001;2:65-73.

21. Buxton EC, Yao W, Lane NE. Changes in serum receptor activator of nuclear factor-kappa beta ligand, osteoprotegerin, and interleukin-6 levels in patients with glucocorticoid-induced osteoporosis treated with human parathyroid hormone (1-34). J Clin Endocrinol Metab. 2004;89: 3332-3336.

22. Liu Y, Porta A, Peng X, et al. Prevention of glucocorticoid-induced apoptosis in osteocytes and osteoblasts by calbindin-D28k. J Bone Miner Res. 2004;19:479-490.

23. Yun SI, Yoon HY, Jeong SY, Chung YS. Glucocorticoid induces apoptosis of osteoblast cells through the activation of glycogen synthase kinase 3beta. J Bone Miner Metab. 2009;27:140-148.

24. Ohnaka K, Tanabe M, Kawate H, Nawata H, Takayanagi R. Glucocorticoid suppresses the canonical Wnt signal in cultured human osteoblasts. Biochem Biophys Res Commun. 2005;329:177-181.

25. Shi XM, Blair HC, Yang X, McDonald JM, Cao X. Tandem repeat of $\mathrm{C} / \mathrm{EBP}$ binding sites mediates PPARgamma2 gene transcription in glucocorticoid-induced adipocyte differentiation. J Cell Biochem. 2000;76:518-527.

26. Carcamo-Orive I, Gaztelumendi A, Delgado J, et al. Regulation of human bone marrow stromal cell proliferation and differentiation capacity by glucocorticoid receptor and AP-1 crosstalk. J Bone Miner Res. 2010;25:2115-2125.

27. Lane NE, Yao W, Balooch M, et al. Glucocorticoid-treated mice have localized changes in trabecular bone material properties and osteocyte lacunar size that are not observed in placebo-treated or estrogendeficient mice. J Bone Miner Res. 2006;21:466-476.

28. Mizushima N, Levine B, Cuervo AM, Klionsky DJ. Autophagy fights disease through cellular self-digestion. Nature. 2008;451:1069-1075. 
29. Xia X, Kar R, Gluhak-Heinrich, et al. Glucocorticoid-induced autophagy in osteocytes. J Bone Miner Res. 2010;25:2479-2488.

30. Saag KG, Shane E, Boonen S, et al. Teriparatide or alendronate in glucocorticoid-induced osteoporosis. $N$ Engl J Med. 2007;357: 2028-2039.

31. Doggett TA, Swarthout JT, Jefcoat SC Jr, et al. Parathyroid hormone inhibits c-jun $\mathrm{N}$ terminal kinase activity in rat osteoblastic cells by a protein kinase A-dependent pathway. Endocrinology. 2002;143: 1880-1888.

32. Sabatini M, Lesur C, Pacherie M, et al. Effects of parathyroid hormone and agonists of the adenylyl cyclase and protein kinase $\mathrm{C}$ pathways on bone cell proliferation. Bone. 1996;18:59-65.

33. Dobnig H, Turner RT. Evidence that intermittent treatment with parathyroid hormone increases bone formation in adult rats by activation of bone lining cells. Endocrinology. 1995;136:3632-3638.

34. Jilka RL. Molecular and cellular mechanisms of the anabolic effect of intermittent PTH. Bone. 2007;40:1434-1446.

35. Jilka RL, O'Brien CA, Ali AA, Roberson PK, Weinstein RS, Manolagas SC. Intermittent PTH stimulates periosteal bone formation by actions on post-mitotic preosteoblasts. Bone. 2008;44:275-286.

36. Jilka RL, Weinstein RS, Bellido T, Roberson P, Parfitt AM, Manolagas SC. Increased bone formation by prevention of osteoblast apoptosis with parathyroid hormone. J Clin Invest. 1999;104:439-446.

37. Datta NS, Pettway GJ, Chen C, Koh AJ, McCauley LK. Cyclin D1 as a target for the proliferative effects of PTH and PTHrP in early osteoblastic cells. J Bone Miner Res. 2007;22:951-964.

38. Canalis E, Giustina A, Bilezikian JP. Mechanisms of anabolic therapies for osteoporosis. N Engl J Med. 2007;357:905-916.

39. Kakar S, Einhorn TA, Vora S, et al. Enhanced chondrogenesis and Wnt signaling in PTH-treated fractures. $J$ Bone Miner Res. 2007;22:1903-1912.

40. Bodine PV, Seestaller-Wehr L, Kharode YP, Bex FJ, Komm BS. Bone anabolic effects of parathyroid hormone are blunted by deletion of the Wnt antagonist secreted frizzled-related protein-1. J Cell Physiol. 2007;210:352-357.

41. Yao W, Cheng Z, Pham A, et al. Glucocorticoid-induced bone loss can be reversed by the actions of PTH and risedronate on different pathways for bone formation and mineralization. Arthritis Rheum. 2008;58:3485-3497.

42. Devogelaer JP, Adler RA, Recknor C, et al. Baseline glucocorticoid dose and bone mineral density response with teriparatide or alendronate therapy in patients with glucocorticoid-induced osteoporosis. J Rheumatol. 2010;37:141-148.

43. Saag KG, Zanchetta JR, Devogelaer JP, et al. Effects of teriparatide versus alendronate for treating glucocorticoid-induced osteoporosis: thirty-six-month results of a randomized, double-blind, controlled trial. Arthritis Rheum. 2009;60:3346-3355.

44. Langsdahl BL, Marin F, Shane E, et al. Teriparatide versus alendronate for treating glucocorticoid-induced osteoporosis:an analysis by gender and menopausal status. Osteoporos Int. 2009;20:2095-2104.

45. Losada BR, Zanchetta FR, Zerbini C, et al. Active comparator trial of teriparatide vs alendronate for treating glucocorticoid-induced osteoporosis: results from the Hispanic and non-Hispanic cohorts. J Clin Densitom. 2009;12:63-70.

46. Gluer CC, Marin F, Ringe JD, et al. Comparative effects of teriparatide and risedronate in glucocorticoid-induced osteoporosis in men: 18 month results of the EuroGIOPS trial. J Bone Miner Res. Epub January 15, 2013.
47. World Health Organization Collaborating Centre for Metabolic Bone Diseases. FRAX: WHO fracture risk assessment tool. Available from: http://www.shef.ac.uk/FRAX. Accessed February 21, 2013.

48. Hansen KE, Wilson HA, Zapalowski C, Fink HA, Minisola S, Adler RA. Uncertainties in the prevention and treatment of glucocorticoid-induced osteoporosis. J Bone Miner Res. 2011;26:1989-1996.

49. [No authors listed]. Recommendations for the prevention and treatment of glucocorticoid-induced osteoporosis: 2001 update. American College of Rheumatology Ad Hoc Committee on Glucocorticoid-Induced Osteoporosis. Arthritis Rheum. 2001;44:1496-1503.

50. Gold DT, Weinstein DL, Pohl G, Krohn KD, Chen Y, Meadows ES. Factors associated with persistence with teriparatide therapy: results from the DANCE observational study. J Osteoporos. 2011;2011:314970.

51. Miller PD, Silverman SL, Gold DT, Taylor KA, Chen P, Wagman RB. Rationale, objectives and designs of the Direct Analysis of Nonvertebral Fracture in the Community Experience (DANCE) study. Osteoporos Int. 2006;17:85-90.

52. Brod M, Rousculp M, Cameron A. Understanding compliance issues for daily self-injectable treatment in ambulatory care settings. Patient Prefer Adherence. 2008;2:129-136.

53. Franceschetti P, Ambrosio M, Zatelli M, et al. Efficacy, safety and adherence to treatment of teriparatide: an observational study. Endocr Abstr. 2012;29:190.

54. Ziller V, Zimmermann SP, Kalder M, et al. Adherence and persistence in patients with severe osteoporosis treated with teriparatide. Curr Med Res Opin. 2010;26:675-681.

55. Hazel-Fernandez L, Louder AM, Foster SA, Uribe CL, Burge RT. Association of teriparatide adherence and persistence with clinical and economic outcomes in Medicare Part D recipients: a retrospective cohort study. BMC Musculoskelet Disord. 2013;14:4.

56. Foster SA, Foley KA, Meadows ES, et al. Adherence and persistence with teriparatide among patients with commercial, Medicare and Medicaid insurance. Osteoporos Int. 2011;22:551-557.

57. Halpern R, Becker L, Iqbal SU, Kazis LE, Macarios D, Badamgarav E. The association of adherence to osteoporosis therapies with fracture, all-cause medical costs, and all-cause hospitalizations: a retrospective claims analysis of female health plan enrollees with osteoporosis. J Manag Care Pharm. 2011;17:25-39.

58. Solomon DH, Katz JN, Jacobs JP, La Tourette AM, Coblyn J. Management of glucocorticoid-induced osteoporosis in patients with rheumatoid arthritis. Arthritis Rheum. 2002;46:3136-3142.

59. Naunton M, Peterson GM, Jones G, Griffin GM, Bleasel MD. Multifaceted educational program increase prescribing of preventive medication for corticosteroid induced osteoporosis. J Rheumatol. 2004;31:550-556.

60. Solomon DH, Katz JN, La Tourette AM, Coblyn JS. Multifaceted intervention to improve rheumatologists' management of glucocorticoidinduced osteoporosis: a randomized controlled trial. Arthritis Rheum. 2004;51:383-387.

61. Newman ED, Matzko CK, Olenginski TP, et al. Glucocorticoid-Induced Osteoporosis Program (GIOP): a novel, comprehensive and highly successful care program with improved outcomes at 1 year. Osteoporos Int. 2006;17:1428-1434.

62. Eli Lilly. Forteo. 2011. Available from: http://www.forteo.com. Accessed February 21, 2013.

63. Teitelbaum SL, Seton MP, Saag KG. Should bisphosphonates be used for long-term treatment of glucocorticoid-induced osteoporosis? Arthritis Rheum. 2011;63:325-328. 
Patient Preference and Adherence

Dovepress

\section{Publish your work in this journal}

Patient Preference and Adherence is an international, peer-reviewed, open access journal focusing on the growing importance of patient preference and adherence throughout the therapeutic continuum. Patient satisfaction, acceptability, quality of life, compliance, persistence and their role in developing new therapeutic modalities and compounds to

optimize clinical outcomes for existing disease states are major areas of interest. This journal has been accepted for indexing on PubMed Central. The manuscript management system is completely online and includes a very quick and fair peer-review system. Visit http://www.dovepress.com/ testimonials.php to read real quotes from published authors.

Submit your manuscript here: http://www.dovepress.com/patient-preference-and-adherence-journal 